\title{
Emerging Pharmacological Treatments to Prevent Abdominal Aortic Aneurysm Growth and Rupture
}

Rodrigo A. Fraga-Silva ${ }^{1}$, Bram Trachet ${ }^{1,2}$, Nikolaos Stergiopulos ${ }^{1}$

${ }^{1}$ Institute of Bioengineering, Ecole Polytechnique Fédérale de Lausanne, Station 17, BM 5125, CH-1007, Lausanne, Switzerland.

${ }^{2}$ IBITech-bioMMeda, Ghent University-IMinds Medical IT, De Pintelaan 185B, 9000 Gent, Belgium.

Corresponding author: Rodrigo Araujo Fraga-Silva, $\mathrm{PhD}$, Institute of Bioengineering, Ecole Polytechnique Fédérale de Lausanne, Station 17, BM 5115, CH-1007, Lausanne, Switzerland. Phone: (+41) 021.693.1119. Email: Rodrigo.fragasilva@epfl.ch 


\begin{abstract}
Abdominal aortic aneurysm (AAA) is a local expansion of the abdominal aorta wall caused by a complex multifactorial maladaptive vascular remodeling. Despite recent advances in the management of cardiovascular diseases, there currently is no established drug therapy for AAA. Since the probability of death from a ruptured AAA still remains high, preventive elective repair of AAAs larger than $5.5 \mathrm{~cm}$ in luminal diameter is considered the best treatment option. However, perioperative complications are problematic as elective AAA repair comes with numerous intrinsic risks. Impelled by the need of improving AAA therapy, significant efforts have been made to identify pharmacological tools that would slow down AAA enlargement and lower the risk of rupture, thereby reducing the necessity of surgical intervention. In this review, we discuss recent findings addressing molecular targets that could potentially treat AAA, particularly addressing: statins, classical renin angiotensin system (RAS) blockers, the protective arm of RAS, renin inhibitors, tetracyclines, interleukin- $1 \beta$ inhibition, anti-angiogenic agents and urocortins.
\end{abstract}

Keywords: abdominal aortic aneurysm, statins, renin angiotensin system, tetracyclines, interleukin-1 $\beta$ 


\section{Introduction}

Abdominal aortic aneurysm (AAA) is a local enlargement of the arterial wall caused by a complex maladaptive vascular remodeling, which affects a large portion of the elderly population [1]. AAA development involves different integrated factors such as biomechanical forces, inflammatory cell infiltrates and the production and activation of various proteases and cytokines. Nevertheless the underlying mechanisms are poorly known [2].

In an early stage of AAA the elastic fibers are degraded by proteases produced by vascular smooth muscle cells and infiltrating macrophages [3]. Consequently, inflammatory cytokines are released leading to an increase in leukocyte infiltration and intensification of proteolytic enzymes production, which potentiates the extracellular matrix disruption [2]. In addition to this process, smooth muscle cells are depleted, contributing to arterial wall weakening $[2,4$, 5]. Moreover, a chronic mural thrombus might be formed, intensifying the inflammatory process and contributing to disease progression [2]. These structural changes in the arterial wall do not only lead to the local enlargement of the vessel, but may also cause its rupture when the arterial wall can no longer withstand the arterial blood pressure $[1,5]$.

Despite recent advances in the management of cardiovascular diseases, there is currently no established drug therapy for AAA, and preventive elective repair of AAAs larger than $5.5 \mathrm{~cm}$ in luminal diameter is considered as the best option since the probability of death from rupture still remains significant [6]. Nonetheless, perioperative complications of elective AAA repair are problematic and present considerable risks [7]. Therefore, motivated by the great need of improving AAA therapy, significant efforts have been made to identify new molecular targets and/or pharmacological tools that would slow down the rate of AAA expansion and reduce the risk of rupture [7, 8], thereby reducing the necessity of surgical intervention, which would be a meaningful improvement for the patients. 
Here, we will discuss the current literature that focuses on potential molecular targets and/or pharmacological tools aimed for treating AAA. In particular, we will detail the statins, classical renin angiotensin system (RAS) blockers, the protective arm of RAS, renin inhibitors, tetracyclines, interleukin- $1 \beta$ inhibition, anti-angiogenic agents and Urocortins.

\section{Statins}

Statins are currently used to lower cholesterol levels by inhibiting the enzyme 3-hydroxy-3methylglutaryl coenzyme A reductase, a central enzyme involved in the production of cholesterol [9]. These drugs are extensively used to prevent atherosclerosis progression and to significantly reduce the risk of adverse events [9]. Numerous studies indicate hyperlipidemia as key factor in the development of AAA [10]; however, there is no clear evidence linking this relationship and recent data supports a contradictory view [7]. In spite of this, statins have been indicated as a potential tool to treat AAA based on its pleiotrophic effects on inflammation, oxidative stress and extracellular matrix composition, which causes reduction of AAA, independent of their cholesterol-lowering effect [11]. Steinmetz and coauthors showed that simvastatin suppresses the development of AAA induced by transient elastase perfusion of the abdominal aorta in both normal and hypercholesterolemic mice [12]. The mechanism of action was independent of lipid reduction and involved a decline in matrix metalloproteinase (MMP)-9 expression, an increase in expression of tissue inhibitor of metalloproteinase (TIMP)-1, and preservation of the medial elastin and smooth muscle cells in the aortic wall [12]. Similarly, simvastatin was shown to prevent and reduce AAA growth in a rabbit model based on aorta exposition to $\mathrm{CaCl}_{2}$ [13]. These beneficial effects were associated with a reduction in MMP-2 and MMP-9 expression [13]. Nagashima and coworkers examined the effect of cerivastatin on MMP-9 production in the aneurysmal wall organ isolated from patients with infrarenal AAA. They found that cerivastatin significantly 
reduced the tissue levels of both total and active MMP-9 in a concentration-dependent manner [14]. Using aortic biopsies from patients that underwent elective open repair of AAA, simvastatin given pre-operatively reduced MMP-9 levels in the aneurysmal wall [15]. Interestingly, in a cohort of 150 patients monitored over 3 years, an association was observed between statin use and a decrease in expansion rate of infrarenal aortic aneurysms [16]. Similarly, other cohorts also reported a decrease in AAA growth following treatment with statins $[17,18]$.

Contrary to these data, no association was reported between prescription statin and AAA expansion in a cohort of 652 patients undergoing surveillance of small AAA [19]. Moreover, different meta-analyses have achieved inconsistent outcomes regarding the beneficial actions of statins on AAA [20-22].

Overall several studies suggest that statins are promising drugs for the treatment of AAA, but further controlled randomized studies are needed to establish a more complete understanding of the effect of statins in AAA treatment.

\section{Renin Angiotensin System - Classical System}

The renin angiotensin system (RAS) is a major regulator of the cardiovascular system and has critical role in the pathophysiology of several diseases, including AAA [5, 23]. Angiotensin (Ang) II, the main RAS effector, is fundamentally important for cardiovascular homeostasis, but its hyperactivity is associated with several cardiovascular diseases [24, 25]. Ang II is mainly formed by the angiotensin-converting enzyme (ACE) and invokes deleterious actions, mostly via activation of the $\mathrm{AT}_{1}$ receptor $[23,24]$. Remarkably, Ang II infusion in lowdensity lipoprotein receptor $\left(\mathrm{Ldlr}^{--}\right)$or apolipoprotein $\mathrm{E}\left(\mathrm{ApoE}^{-{ }_{-}}\right)$gene deleted mice is sufficient to induce AAA-like dilatation of the abdominal aorta [26-28] (re-defined as 
dissecting abdominal aortic aneurysm [29, 30]), highlighting a central role of Ang II in the pathogenesis of AAA [5]. Based on these and other observations, the pharmacological blockage of Ang II, by inhibition of angiotensin-converting enzyme (ACE) or blockage of $\mathrm{AT}_{1}$ receptors, has emerged as a potential tool to prevent AAA growth and/or reduce the risk of rupture [5]. In different studies, Ang II-induced dissecting AAA formation in $\mathrm{ApoE}^{-/}$mice was prevented by $\mathrm{AT}_{1}$ receptor blockers $(\mathrm{ARBs})$ or $\mathrm{ACE}$ inhibitors (ACEi) [31, 32]. Such outcomes were predictable, especially for ARBs, since the dissecting AAA model was based on Ang II hyperactivity. Nevertheless, the beneficial effects of these drugs against dissecting AAA were not restricted to the Ang II-induced model. ARBs and ACEi significantly ameliorated AAA in different animal models, including AAA-induction by intra-aortic infusion of porcine pancreatic elastase and in a DOCA-salt model associated to degeneration of elastic lamina by b-aminopropionitrile infusion, both of which are AAA models independent of Ang II infusion [33-36].

Ang II induces dissecting AAA by modulating different molecular mechanisms. Interestingly, Ang II provokes dissecting AAA in hypercholesterolemic mice, independent of increases in blood pressure [37]. In the early stage of dissecting AAA development, Ang II has been shown to contribute to infiltration and accumulation of macrophages in the sub intima, mainly by stimulating CCL2 (also known as MCP-1) secretion from endothelial and vascular smooth muscle cells [38-40]. Additional inflammatory pathways are also involved in the action of Ang II. Some evidence has suggested that CCL5 also has an important role in Ang II induced dissecting AAA [41]. Moreover, Ang II may stimulate the expression of cyclooxygenase-2 (COX-2) [42, 43], a critical enzyme in AAA pathogenesis that forms vasoactive and inflammatory prostaglandins. Indeed, it has been shown that COX-2 deficiency attenuates Ang II infusion-mediated dissecting AAA [44]. Ang II also may stimulate osteopontin (OPN) secretion and actions [45]. OPN is a secreted extracellular 
structural protein with pleiotropic functions that have been implicated in tissue repair, remodeling, and inflammation which also has an important role in AAA [45]. OPN acts as a chemotactic cytokine and modulates adhesion, migration, and activation of macrophages [46].

Ang II also intensifies dissecting AAA evolution in mice by stimulating the production and activity of MMPs with consequent degradation of the arterial wall integrity [47, 48]. Indeed, doxycycline, a broad specificity inhibitor of MMPs, attenuates the incidence and severity of dissecting AAA induced by Ang II infusion [49]. Similarly, MMP-2-deficiency is known to inhibit Ang II induced elastin degradation in ApoE-/- mice [50].

Another important mechanism triggered by Ang II stimulus is the hyper-production of oxidative stress [51]. Numerous studies have demonstrated Ang II as a potent stimulator of reactive oxygen species (ROS) in a variety of disorders [23]. ROS overproduction leads to an increased inflammatory profile, MMP activation and smooth muscle cell apoptosis, potentiating AAA development [52]. Interestingly, treatment with vitamin E, an antioxidant agent, ameliorates dissecting AAA formation in Ang II-infused ApoE-/- mice [53]. Accordingly, it was shown that Ang II-derived ROS triggers nuclear translocation of the AMP-activated protein kinase, a central energy-sensing enzyme that monitors cellular energy status, resulting in aberrant expression of MMP2, extracellular matrix degradation and consequent AAA worsening [50].

Taken together, the evidence strongly suggest that RAS acts as a key player in the pathogenesis of dissecting AAA in mice, suggesting that compounds that target this system could be potential tools to inhibit AAA progression and/or prevent AAA rupture. However, most information is based on angiotensin II-infused mice which show dissection-like features that are not reproduced in human AAA [29, 38] . Information from clinical trials is limited, especially regarding the effectiveness of ACEi and ARBs. The latter is indispensable if and 
when such drugs were to be considered for clinical treatment of AAA. A new clinical trial might, however, provide these critical data. In an ongoing phase 4 study, the effectiveness of Telmisartan in slowing down the progression of abdominal aortic aneurysms and reducing the circulating concentrations of AAA biomarkers is being investigated in comparison to placebo (ClinicalTrials.gov number NCT01683084).

\section{The Protective Arm of RAS - ACE2/Ang-(1-7)/Mas receptor}

In the classical paradigm, the RAS actions are triggered by a sequence of enzymatic reactions that is generated by Ang II. This enzymatic cascade is initiated by renin, which breaks down the precursor angiotensinogen to Ang I, an inactive decapeptide. Sequentially, ACE cleaves Ang I and forms the octapeptide Ang II. Ang II exerts its biological effects via activation of two distinct G protein-coupled receptors, AT1 and AT2, but the deleterious actions of Ang II are mediated by AT1 receptors [23]. In fact, the relevance of Ang II/AT1 is evidenced by the remarkable success obtained by drugs based on the pharmacological inhibition of ACE and blockage of AT1 receptors, which are frontline treatment options for hypertension. Contrary to the $\mathrm{AT}_{1}$ receptor, activation of the $\mathrm{AT}_{2}$ receptor appears to provide beneficial actions, such as vasodilation, antiproliferative and anti-inflammatory actions, thus counter-regulating the $\mathrm{AT}_{1}$ receptor effects [54-56]. Despite the fact that actions triggered by $\mathrm{AT}_{1}$ prevail over counter-actions triggered by $\mathrm{AT}_{2}$, the stimulation of the $\mathrm{AT}_{2}$ receptor apparently contributes to the beneficial actions produced by $\mathrm{AT}_{1}$ receptor blockers, suggesting that selective $\mathrm{AT}_{2}$ agonists may be used as potential tools for the treatment of cardiovascular disease [54,57], including AAA $[31,58]$. Indeed, there is evidence that $\mathrm{AT}_{2}$ activation produces beneficial anti-AAA outcomes. For instance, co-infusion of Ang II and PD123319 (an AT2 receptor antagonist) in ApoE-/- mice significantly worsened AAA, suggesting a protective action mediated by AT2 [31]. Similarly, AT2-gene deletion enhances the abnormal growth and 
rupture of the aorta in a mouse model of Marfan syndrome [58]. Contrarily, other reports have shown that AT2 deficiency does not affect Ang II-induced AAA in $\mathrm{LDL}^{-/-}$mice while PD123319 augments AAA through a mechanism that is AT2 receptor independent [59]. Such conflicting data could be explained by the different backgrounds of the used animals and the poor selectivity of PD123319, since this compound can also block the MrgD receptor for alamandine [60].

Taken together, emerging data suggest that selective AT2 agonists may be effective for treating AAA. However, due to the conflicting and limited data, the role of AT2 on AAA and efficacy of selective AT2 agonists must be further evaluated.

In 2000, an important component of the RAS has been discovered, the angiotensin converting enzyme 2 (ACE2) [61, 62]. This enzyme breaks down Ang II to form Ang-(1-7), a heptapeptide with actions often contrary to those of Ang II [63-65]. Ang-(1-7), which binds to its own receptor, the Mas GPCR, induces many protective actions, such as vasodilation, reduction of oxidative stress, and anti-inflammatory and antithrombotic effects $[64,66]$. Therefore, ACE2 is a key player that regulates the balance of an Ang II and Ang-(1-7), a deleterious and protective effector $[64,66]$. Based on that, ACE2 has emerged as a potential target to treat cardiovascular diseases, including AAA. Thatcher and co-workers have shown that ACE2 is detectable in murine and human AAAs [67]. Moreover, they observed that ACE2 deficiency worsens dissecting AAA in the mouse model based on Ang II infusion [67]. Interestingly, diminazene aceturate, an ACE2 activator compound, ameliorates and markedly reduces the incidence of dissecting AAA in Ang II-infused mice, suggesting that ACE2 could be a potential target in the treatment of AAA and identifies diminazene as a possible pharmacological tool [67]. Despite these exciting findings, the potential of ACE2 and effectivity of diminazene or other ACE2 activator compounds must be confirmed. 


\section{Renin Inhibition}

Beyond the previously mentioned strategies that target RAS, another key component of this system has been developed. Aliskiren is the first representative of a new class drugs based on the inhibition of renin, thereby focusing on an upstream RAS mediator [68]. Recently, Seto and co-authors showed that aliskiren significantly inhibits the expansion of the dissecting AAA diameter in the Ang II-infused $\mathrm{ApoE}^{-/-}$mouse model, as assessed by ultrasound [69]. This effect was associated with a reduction in T-lymphocytes and macrophages infiltration in the aorta wall [69]. Moreover, it caused a reduction in pro-renin receptor expression and mitogen-activated protein kinase (MAPK) activity in the suprarenal aorta [69]. A clinical trial aimed at addressing the anti-inflammatory effect of aliskiren in patients with small AAA and mild hypertension was initiated at the end of 2011; however, the trial was terminated due to an insufficient number of participants (ClinicalTrials.gov number NCT01425242). Overall, renin inhibition appears to present interesting outcomes against AAA; however, the necessary scientific support remains limited.

\section{Tetracycline}

The modulation of extracellular matrix composition is fundamental in the maintenance of the vascular wall integrity [47]. MMPs are the major enzymes in the vascular remodeling, and over-activity of such enzymes is closely associated to AAA pathogenesis and progression $[47,70]$. Based on the relevance of MMPs on AAA, doxycycline emerged as a potential tool for the treatment of AAA. This antibiotic agent, member of the tetracycline antibiotic class, is listed as one of the most important medications needed for basic health in the World Health Organization's List of Essential Medicines. However, its protective effects against AAA are not related to its antibiotic activity, but to the inhibition of MMPs [8]. The effectivity of 
doxycycline against AAA was first shown in a rat model of AAA, induced by elastase perfusion. Doxycycline treatment reduced the incidence and diameter of AAA, which was associated with a reduction in aortic elastin degradation without altering the influx of inflammatory cells [71]. Doxycycline was also effective in inhibiting AAA growth in other AAA animal models, such as aneurysm induced by bathing the periadventitial aortic with $\mathrm{CaCl}_{2}$ [72] or dissecting AAA induced by Ang II infusion in ApoE-/- mice [49, 73]. It has been shown that chemically modified tetracyclines without antibiotic activity exhibited similar efficacy to that of doxycycline by inhibiting AAA growth and disrupting the medial elastin in a rat model of AAA, induced by elastase perfusion [74]. Contradictory to these studies, Lida et al reported that doxycycline does not influence aneurysm progression in two different AAA animal models, Ang II-infused $\mathrm{ApoE}^{-/-}$mice or transient intra-aortic porcine pancreatic elastase infusion in B6 mice [33]. Similarly, Xie showed that doxycycline did not affect dissecting AAA progression and aortic rupture in Ang II-infused mice [75].

To better evaluate the effects of doxycycline against AAA, a number of studies have addressed the action of doxycycline in human AAA. It has been documented that preoperative doxycycline treatment (for 7 days) in patients who underwent elective repair of an infrarenal AAA, reduced MMP-9 expression and post-translational processing of MMP-2 in aneurysmal tissues [76]. Interestingly, in a prospective phase II study (cohort $n=36$ ), Baxter and co-workers demonstrated that prolonged treatment (6 month) with doxycycline promoted a gradual reduction of MMP-9 plasma levels compared to baseline levels in patients with small asymptomatic AAAs [77]. In a randomized, double-blind, placebocontrolled pilot study, the effectivity of doxycycline was evaluated in patients (cohort $n=32$ ) with AAA growth larger than $30 \mathrm{~mm}$ but less than $55 \mathrm{~mm}$. The aneurysm expansion rate in the doxycycline group was significantly lower than in the placebo group during the 6-12 month and 12-18 month period [78]. However, a large imbalance in the randomization of 
smoking patients (smoking patients: doxycycline group 3/17, 17.6\%; placebo group 8/15, $53.3 \%,[78]$ ) may represent an important concern for the interpretation of these results, due to the fact that smoking is one of the most prevalent risk factors for AAA development and progression [79].

Most of the reports focused on the expression/activity of MMP-9 and MMP-2 when evaluating the actions of doxycycline on AAA [80]; moreover, other MMPs types and additional mechanism appear to be involved. Apparently, doxycycline also reduces the expression of MMP-3 and MMP-25 in aortic aneurysmal tissue [81]. Furthermore, this compound enhances Cystatin C and TIMP-1, important MMP inhibitors [81]. Beyond regulating the major extracellular matrix modulators, doxycycline also produced a significant and selective suppression of aortic wall inflammation by reducing neutrophil infiltration, as well as cytotoxic T-cell and vascular cytokines [82].

Contrarily to these studies, a recent randomized clinical trial did not observe any influence of doxycycline on small AAAs [83]. In a cohort of 286 patients, which represents a larger database than previous studies, small AAAs were monitored under doxycycline or placebo treatment for 18 months. Surprisingly, an increased aneurysm growth in patients receiving doxycycline was observed, while the need of elective surgery was similar between the doxycycline and the placebo group [83]. These controversial data may be explained by the different doses used in the studies (Mosorin et at 150mg/day [78] ; Meijer et al 100mg/day [83]). An ongoing randomized controlled double-blind clinical trial using a higher dose (100 mg twice a day) may provide additional information regarding the effectivity of doxycycline. 


\section{Interleukin-1}

Interleukin-1 (IL-1) is a key cytokine in the inflammatory cascade [84] and is a central factor and powerful modulator of AAA genesis and progression [85-87]. Production of IL1 $\beta$ was significantly higher in aneurysmal tissues than control aortic tissue from cadaveric donors [87]. Similarly, the circulating IL1 $\beta$ was markedly increased in patients with AAA [86]. These findings strongly suggest that IL $1 \beta$ is involved in the pathogenesis of AAA. In fact, Johnston et al demonstrated that IL-1 $\beta$ expression was elevated in the abdominal aorta of B6 mice after elastase aortic perfusion [85]. Moreover, IL1 $\beta$ - or IL1R-gene deficiency protected against elastase perfusion-induced AAA formation, which is associated with a reduction in macrophage and neutrophil infiltration, as well as elastin preservation [85]. In the same study, pretreatment with Anakinra, an IL1R antagonist, significantly inhibited AAA enlargement, macrophage infiltration and elastin disruption [85]. Together, these findings suggest IL1 $\beta /$ IL1R is a potential target for the pharmacological treatment of AAA. Currently, a multicenter, randomized, double-blind, placebo-controlled phase II clinical trial (ClinicalTrials.gov number NCT02007252) aims to assess the safety, tolerability and efficacy of ACZ885 on aneurysmal growth rate in 100 patients with AAA. ACZ885, named Canakinumab, is a human monoclonal antibody targeted at interleukin-1 beta. The outcome of this trial may provide valuable insights for the pharmacological treatment for AAA.

\section{Anti-angiogenic therapy}

The use of anti-angiogenic agents for the treatment of AAA has been speculated recently based on evidence demonstrating that exacerbated angiogenesis in human and animal AAA wall may contribute to the initiation and progression of aneurysm [88]. Different studies have shown an intense neovascularization in the aneurysmal aortic walls of human and animal 
models. Holmes et al performed a morphometric analysis in human aortic aneurysmatic tissues and observed an augmented medial neovascularization compared to normal aortas [89]. Similarly, another study documented increased angiogenesis in all layers (media, adventitia and a transition zone) of the human aortic aneurysmal wall. The same study also reported that the degree of neovascularization in the AAA was highly correlated with the amount of the inflammatory cell infiltration [90], suggesting an association between the angiogenesis and cell infiltration. Performing histological analyses in human AAA biopsies, Kobayashi et al observed that the augmented neovascularization in the aneurysmatic walls was associated with augmented vascular endothelial growth factor (VEGF) expression in the infiltrated area [91], suggesting that the neoangiogenesis within the AAA wall is driven by VEGF overexpression, a potent angiogenic factor. In accordance with that, recombinant human VEGF treatment intensified the formation of Ang II-induced dissecting AAAs in ApoE-/- mice [92].

The studies mentioned above point out angiogenesis as a critical mechanism in the pathogenesis of AAA, contributing to the increase of cell infiltration and consequent degeneration of the aortic wall. Additionally, beyond its critical role in the initiation of aneurysm, evidence indicates that angiogenesis also modulates the ongoing process of mature aortic aneurysm, contributing to the expansion and rupture of aortic wall [93]. Indeed, Choke et al found that biopsy samples from aneurysm rupture edge displayed increased medial neovascularization compared to the aneurysm wall at the level of rupture (region $3 \mathrm{~cm}$ away from the rupture edge) and from the anterior sac. Moreover, they observed that the aneurysm rupture edge displayed an increased expression of proangiogenic cytokines ( $\alpha$ v-integrin, VEGF, VE-cadherin, MCP-1, and vimentin) [94]. Thus the focal increase in medial neovascularization in the aneurysm rupture edge, with a concomitant increase in 
proangiogenic expression, may contribute to the aneurysm rupture; however, further investigations are necessary to confirm this hypothesis.

The use of anti-angiogenic drugs has been approved for the treatment of numerous diseases. In fact, this drug class is a landmark for the treatment of different modalities of cancer [95]. Based on the critical role of angiogenic pathways in human AAAs, anti-angiogenic drugs may therefore also represent a potential tool to reduce the progression of the aneurysms and diminish the risk rupture. In this line, VEGF, one of the most potent angiogenic and vascular permeability factors, has been pointed out by different groups as a potential target for such compounds against AAA. Evidence has shown a critical role of VEGF in the pathophysiology of AAA. For instance, it was documented that the intensity of angiogenesis in human aortic aneurysmal was associated to a larger amount of infiltrated inflammatory cells expression VEGF [91]. Moreover, recombinant human VEGF treatment increased the formation of Ang II-induced aneurysms in ApoE-/- mice [89, 96]. In accordance, treatment with the soluble form of VEGF-A receptor-1 (negative modulator of VEGF) reduced aneurysm size, restored wavy structure of the elastic lamellae, reduced cell infiltration, attenuated MMP activity and decreased neoangiogenesis in an animal model of AAA induced by periaortic application of $\mathrm{CaCl}_{2}$ [96]. Together, these results suggest that pharmacological compounds able to block VEGF may be beneficial for AAA treatment. Currently, drugs that block VEGF actions are readily available in the clinic. These drugs are used in the treatment of certain cancers and in age-related macular degeneration. However, so far, no study has explored the action of these drugs against AAA. Controversially, a case report has inferred that intravitreal injection of bevacizumab, a recombinant monoclonal anti-VEGF-A, was associated with AAA rupture $\{$ Baek, $2014 \# 105\}$. While this is of course a single case, this evidence calls for further investigation. 


\section{Urocortin 2}

Urocortins (UCN) are hormones members of the corticotropin-releasing hormone family that have an essential role in the central system and peripheral tissues [97]. In the last years, UCNs emerged as critical modulators of the cardiovascular system and relevant efforts have been engaged to explore its peripheral actions [97]. These endogenous hormones are peptides with 38 to 40 aminoacids that act through activation of two distinguished G-protein-coupled receptors (GPCR), corticotrophin-releasing factor receptor (CRFR) type 1 and type 2 . Three isoforms of UNCs have been described, UCN1, UCN2 and UCN3; interestingly, they exhibit different affinity to the CRFRs. While UCN1 binds to both CRFR1 and CRFR2, UCN2 and UCN3 show selectively binding to the CRFR2 [98]. CRFR1 and CRFR2 are expressed in the central system and periphery. However, the actions of CRFR2 predominate in the cardiovascular system $[97,98]$.

Various studies in humans and animals have shown the implication of UCNs in different cardiovascular disorders, such as atherosclerosis, myocardial infarct and heart failure [97, 99, 100]; however, its role on AAA has only been reported very recently [101, 102]. The first evidence associating the UCNs to AAA was documented by Rush et al [102]. Using the Ang II-infused ApoE-/- mouse AAA model, they investigated genes that were differentially expressed in the aneurysm prone segment of aorta. Interestingly, UCN3 gene expression was downregulated in the suprarenal region of the aorta (susceptive to aneurysm formation in mice) compared to the infrarenal region (resistant to aneurysm formation in mice)[102], raising the speculation that UCNs have a protective function against AAA. In another study, the same group reported that UCN2 and CRFR2 gene expression were significantly higher in the most dilated part of the human AAA compared to the non-dilated proximal neck of the AAA [101]. Moreover, in a well-controlled cohort they also documented that UCN2 plasma level was higher in AAA patients compared to non-aneurysmal patients [89]. Interestingly, 
UCN2 protein expression within the AAA wall was mostly co-located with infiltrating Tlymphocytes and neutrophils [101], suggesting that these infiltrated immune cells are the source of UCN2 production within the AAA wall. In keeping with that, it was observed that explant preparation from the dilated portion of AAA releases larger amount of UCN2 compared to non-dilated proximal neck of the AAA [101]. These data demonstrate that UCN2 has a plausive role on AAA pathogenesis; however, do not provide information about its function, if beneficial or detrimental.

In the cardiovascular system, UCNs have been shown to be key modulators of inflammation and apoptosis, critical mechanisms in AAA pathology. For instance, it was shown that proinflammatory cytokines, such as TNF $\alpha$ and IFN $\gamma$, upregulated UCN1 protein expression in human umbilical vein endothelial cells, while UCN1 effectively attenuated the generation of ROS induced by Ang II [103]. In another study, it was found that UCN1 suppressed the LPSinduced upregulation of MCP1 and ICAM1 in human endothelial cells [100]. Moreover, in human aortic smooth muscle cells, UCN1 suppressed Ang II-induced migration, inhibit cell proliferation without inducing apoptosis and enhance the activities of MMP2 and MMP9 [100]. Others reported that UCN1 treatment inhibited cell proliferation and VEGF release in rat smooth muscle cells [104]. Accordingly, UCN2 decreased human vascular smooth muscle proliferation and increases IL-6 secretion in a dependent manner of CRFR2 [101]. Taken together these data indicate that UNCs modulate critical molecular pathways of AAA pathophysiology in a protective manner; however, the speculation about the potential of UNCs against AAA must be evaluated in future studies.

\section{Perspectives and Future Directions}

Overall, the discussion above demonstrates that there are no satisfactory data clearly indicating to a single pharmacological approach for the treatment of AAA. Moreover, 
evidence points to emerging targets for which the real potential needs to be investigated. The following key topics should be considered:

1) Despite the fact that statins and tetracyclines have been suggested by several preclinical and clinical trials as interesting tools to attenuate AAA growth and rupture probability, conflicting data hamper the recommendation of such drugs in clinical practice. Therefore, large and well-controlled clinical trials are needed to assess the effectiveness of these drugs;

2) Classical RAS blockers (ARBs and ACEi) appear to be promising agents in the treatment of AAA; however, the literature addressing the effectivity of ACEi and ARBs in human AAA is limited, and clinical trials are needed to assess its potential;

3) The protective arm of the RAS (ACE2/Ang-(1-7)/Mas receptor), renin inhibitors, pharmacological inhibition of IL-1 and anti-angiogenic agents have emerged as potential molecular targets against AAA, however the mechanisms of action and effectivity of these targets must be further assessed;

4) Few studies suggest UCNs have an important role on AAA pathophysiology, and its beneficial effects against AAA may arise from its anti-inflammatory and -antiapoptotic actions in the cardiovascular system. However, the number of published data is limited and the contribution of UNC, if benefic or detrimental, is still not clear shown. Further studies are needed to evaluate the direct actions of UCNs on AAA and assess its relevance and potential as a pharmacological tool to treat AAA.

\section{Conclusion}

Significant efforts have been made to identify new molecular targets able to retard AAA progression and reduced the risk of rupture. So far, there are no satisfactory data that clearly 
point out to a specific treatment. Recently, promising candidates have been suggested but they required further investigation to assess its real potential.

\section{Conflict of Interest}

None to be declared 


\section{References}

1. Michel, J.B., et al., Novel aspects of the pathogenesis of aneurysms of the abdominal aorta in humans. Cardiovasc Res, 2011. 90(1): p. 18-27.

2. Sakalihasan, N., R. Limet, and O.D. Defawe, Abdominal aortic aneurysm. Lancet, 2005. 365(9470): p. 1577-89.

3. Longo, G.M., et al., Matrix metalloproteinases 2 and 9 work in concert to produce aortic aneurysms. J Clin Invest, 2002. 110(5): p. 625-32.

4. Thompson, R.W., S. Liao, and J.A. Curci, Vascular smooth muscle cell apoptosis in abdominal aortic aneurysms. Coron Artery Dis, 1997. 8(10): p. 623-31.

5. Malekzadeh, S., et al., Role of the renin-angiotensin system on abdominal aortic aneurysms. Eur J Clin Invest, 2013. 43(12): p. 1328-38.

6. Lederle, F.A., et al., Immediate repair compared with surveillance of small abdominal aortic aneurysms. N Engl J Med, 2002. 346(19): p. 1437-44.

7. Baxter, B.T., M.C. Terrin, and R.L. Dalman, Medical management of small abdominal aortic aneurysms. Circulation, 2008. 117(14): p. 1883-9.

8. Kurosawa, K., J.S. Matsumura, and D. Yamanouchi, Current status of medical treatment for abdominal aortic aneurysm. Circ J, 2013. 77(12): p. 2860-6.

9. Prospective Studies, C., et al., Blood cholesterol and vascular mortality by age, sex, and blood pressure: a meta-analysis of individual data from 61 prospective studies with 55,000 vascular deaths. Lancet, 2007. 370(9602): p. 1829-39.

10. Manning, M.W., et al., Abdominal aortic aneurysms: fresh insights from a novel animal model of the disease. Vasc Med, 2002. 7(1): p. 45-54.

11. Mihos, C.G., M.J. Salas, and O. Santana, The pleiotropic effects of the hydroxymethyl-glutaryl-CoA reductase inhibitors in cardiovascular disease: a comprehensive review. Cardiol Rev, 2010. 18(6): p. 298-304.

12. Steinmetz, E.F., et al., Treatment with simvastatin suppresses the development of experimental abdominal aortic aneurysms in normal and hypercholesterolemic mice. Ann Surg, 2005. 241(1): p. 92-101.

13. Mastoraki, S.T., et al., Treatment with simvastatin inhibits the formation of abdominal aortic aneurysms in rabbits. Ann Vasc Surg, 2012. 26(2): p. 250-8.

14. Nagashima, H., et al., A 3-hydroxy-3-methylglutaryl coenzyme A reductase inhibitor, cerivastatin, suppresses production of matrix metalloproteinase-9 in human abdominal aortic aneurysm wall. J Vasc Surg, 2002. 36(1): p. 158-63.

15. Evans, J., et al., Simvastatin attenuates the activity of matrix metalloprotease-9 in aneurysmal aortic tissue. Eur J Vasc Endovasc Surg, 2007. 34(3): p. 302-3. 
16. Schouten, O., et al., Statins are associated with a reduced infrarenal abdominal aortic aneurysm growth. Eur J Vasc Endovasc Surg, 2006. 32(1): p. 21-6.

17. Schlosser, F.J., et al., Growth predictors and prognosis of small abdominal aortic aneurysms. J Vasc Surg, 2008. 47(6): p. 1127-33.

18. Sukhija, R., et al., Mortality and size of abdominal aortic aneurysm at long-term follow-up of patients not treated surgically and treated with and without statins. Am J Cardiol, 2006. 97(2): p. 279-80.

19. Ferguson, C.D., et al., Association of statin prescription with small abdominal aortic aneurysm progression. Am Heart J, 2010. 159(2): p. 307-13.

20. Takagi, H., et al., Effects of statin therapy on abdominal aortic aneurysm growth: a meta-analysis and meta-regression of observational comparative studies. Eur J Vasc Endovasc Surg, 2012. 44(3): p. 287-92.

21. Twine, C.P. and I.M. Williams, Systematic review and meta-analysis of the effects of statin therapy on abdominal aortic aneurysms. Br J Surg, 2011. 98(3): p. 346-53.

22. Sweeting, M.J., et al., Meta-analysis of individual patient data to examine factors affecting growth and rupture of small abdominal aortic aneurysms. Br J Surg, 2012. 99(5): p. 655-65.

23. Bader, M., Tissue renin-angiotensin-aldosterone systems: Targets for pharmacological therapy. Annu Rev Pharmacol Toxicol, 2010. 50: p. 439-65.

24. Matsusaka, T. and I. Ichikawa, Biological functions of angiotensin and its receptors. Annu Rev Physiol, 1997. 59: p. 395-412.

25. de Gasparo, M., et al., International union of pharmacology. XXIII. The angiotensin II receptors. Pharmacol Rev, 2000. 52(3): p. 415-72.

26. Daugherty, A., M.W. Manning, and L.A. Cassis, Angiotensin II promotes atherosclerotic lesions and aneurysms in apolipoprotein E-deficient mice. J Clin Invest, 2000. 105(11): p. 1605-12.

27. Daugherty, A. and L. Cassis, Chronic angiotensin II infusion promotes atherogenesis in low density lipoprotein receptor -/- mice. Ann N Y Acad Sci, 1999. 892: p. 108-18.

28. Daugherty, A., L.A. Cassis, and H. Lu, Complex pathologies of angiotensin IIinduced abdominal aortic aneurysms. J Zhejiang Univ Sci B, 2011. 12(8): p. 624-8.

29. Trachet, B., et al., Dissecting abdominal aortic aneurysm in Ang II-infused mice: suprarenal branch ruptures and apparent luminal dilatation. Cardiovasc Res, 2015. 105(2): p. 213-22.

30. Schriefl, A.J., et al., Remodeling of intramural thrombus and collagen in an Ang-II infusion ApoE-/- model of dissecting aortic aneurysms. Thromb Res, 2012. 130(3): p. e139-46. 
31. Daugherty, A., M.W. Manning, and L.A. Cassis, Antagonism of AT2 receptors augments angiotensin II-induced abdominal aortic aneurysms and atherosclerosis. $\mathrm{Br}$ J Pharmacol, 2001. 134(4): p. 865-70.

32. Inoue, N., et al., Involvement of vascular angiotensin II-forming enzymes in the progression of aortic abdominal aneurysms in angiotensin II- infused ApoE-deficient mice. J Atheroscler Thromb, 2009. 16(3): p. 164-71.

33. Iida, Y., et al., Efficacy and mechanism of angiotensin II receptor blocker treatment in experimental abdominal aortic aneurysms. PLoS One, 2012. 7(12): p. e49642.

34. Liao, S., et al., Suppression of experimental abdominal aortic aneurysms in the rat by treatment with angiotensin-converting enzyme inhibitors. J Vasc Surg, 2001. 33(5): p. 1057-64.

35. Fujiwara, Y., et al., Inhibition of experimental abdominal aortic aneurysm in a rat model by the angiotensin receptor blocker valsartan. Int J Mol Med, 2008. 22(6): p. 703-8.

36. Kanematsu, Y., et al., Pharmacologically induced thoracic and abdominal aortic aneurysms in mice. Hypertension, 2010. 55(5): p. 1267-74.

37. Cassis, L.A., et al., ANG II infusion promotes abdominal aortic aneurysms independent of increased blood pressure in hypercholesterolemic mice. Am J Physiol Heart Circ Physiol, 2009. 296(5): p. H1660-5.

38. Saraff, K., et al., Aortic dissection precedes formation of aneurysms and atherosclerosis in angiotensin II-infused, apolipoprotein E-deficient mice. Arterioscler Thromb Vasc Biol, 2003. 23(9): p. 1621-6.

39. Daugherty, A., et al., Angiotensin II infusion promotes ascending aortic aneurysms: attenuation by CCR2 deficiency in apoE-/- mice. Clin Sci (Lond), 2010. 118(11): p. 681-9.

40. Ishibashi, M., et al., Bone marrow-derived monocyte chemoattractant protein-1 receptor CCR2 is critical in angiotensin II-induced acceleration of atherosclerosis and aneurysm formation in hypercholesterolemic mice. Arterioscler Thromb Vasc Biol, 2004. 24(11): p. e174-8.

41. Iida, Y., et al., Peptide inhibitor of CXCL4-CCL5 heterodimer formation, MKEY, inhibits experimental aortic aneurysm initiation and progression. Arterioscler Thromb Vasc Biol, 2013. 33(4): p. 718-26.

42. Jaimes, E.A., et al., Up-regulation of glomerular COX-2 by angiotensin II: role of reactive oxygen species. Kidney Int, 2005. 68(5): p. 2143-53.

43. King, V.L., et al., Selective cyclooxygenase-2 inhibition with celecoxib decreases angiotensin II-induced abdominal aortic aneurysm formation in mice. Arterioscler Thromb Vasc Biol, 2006. 26(5): p. 1137-43. 
44. Gitlin, J.M., et al., Genetic deficiency of cyclooxygenase-2 attenuates abdominal aortic aneurysm formation in mice. Cardiovasc Res, 2007. 73(1): p. 227-36.

45. Bruemmer, D., et al., Angiotensin II-accelerated atherosclerosis and aneurysm formation is attenuated in osteopontin-deficient mice. J Clin Invest, 2003. 112(9): p. 1318-31.

46. O'Regan, A. and J.S. Berman, Osteopontin: a key cytokine in cell-mediated and granulomatous inflammation. Int J Exp Pathol, 2000. 81(6): p. 373-90.

47. Freestone, T., et al., Inflammation and matrix metalloproteinases in the enlarging abdominal aortic aneurysm. Arterioscler Thromb Vasc Biol, 1995. 15(8): p. 1145-51.

48. Eagleton, M.J., et al., Early increased MT1-MMP expression and late MMP-2 and MMP-9 activity during Angiotensin II induced aneurysm formation. J Surg Res, 2006. 135(2): p. 345-51.

49. Manning, M.W., L.A. Cassis, and A. Daugherty, Differential effects of doxycycline, a broad-spectrum matrix metalloproteinase inhibitor, on angiotensin II-induced atherosclerosis and abdominal aortic aneurysms. Arterioscler Thromb Vasc Biol, 2003. 23(3): p. 483-8.

50. Wang, S., et al., Activation of AMP-activated protein kinase alpha2 by nicotine instigates formation of abdominal aortic aneurysms in mice in vivo. Nat Med, 2012. 18(6): p. 902-10.

51. Virdis, A., et al., Role of $N A D(P) H$ oxidase on vascular alterations in angiotensin IIinfused mice. J Hypertens, 2004. 22(3): p. 535-42.

52. Satoh, K., et al., Cyclophilin A enhances vascular oxidative stress and the development of angiotensin II-induced aortic aneurysms. Nat Med, 2009. 15(6): p. 649-56.

53. Gavrila, D., et al., Vitamin E inhibits abdominal aortic aneurysm formation in angiotensin II-infused apolipoprotein E-deficient mice. Arterioscler Thromb Vasc Biol, 2005. 25(8): p. 1671-7.

54. Widdop, R.E., et al., AT2 receptor-mediated relaxation is preserved after long-term AT1 receptor blockade. Hypertension, 2002. 40(4): p. 516-20.

55. Steckelings, U.M., et al., The angiotensin AT2 receptor in left ventricular hypertrophy. J Hypertens, 2010. 28 Suppl 1: p. S50-5.

56. Steckelings, U.M., et al., AT2 receptor agonists: hypertension and beyond. Curr Opin Nephrol Hypertens, 2012. 21(2): p. 142-6.

57. Savoia, C., et al., Angiotensin type 2 receptor in hypertensive cardiovascular disease. Curr Opin Nephrol Hypertens, 2011. 20(2): p. 125-32. 
58. Habashi, J.P., et al., Angiotensin II type 2 receptor signaling attenuates aortic aneurysm in mice through ERK antagonism. Science, 2011. 332(6027): p. 361-5.

59. Daugherty, A., et al., PD123319 augments angiotensin II-induced abdominal aortic aneurysms through an AT2 receptor-independent mechanism. PLoS One, 2013. 8(4): p. e61849.

60. Lautner, R.Q., et al., Discovery and characterization of alamandine: a novel component of the renin-angiotensin system. Circ Res, 2013. 112(8): p. 1104-11.

61. Donoghue, M., et al., A novel angiotensin-converting enzyme-related carboxypeptidase (ACE2) converts angiotensin I to angiotensin 1-9. Circ Res, 2000. 87(5): p. E1-9.

62. Vickers, C., et al., Hydrolysis of biological peptides by human angiotensin-converting enzyme-related carboxypeptidase. J Biol Chem, 2002. 277(17): p. 14838-43.

63. Ferrario, C.M., ACE2: more of Ang-(1-7) or less Ang II? Curr Opin Nephrol Hypertens, 2011. 20(1): p. 1-6.

64. Fraga-Silva, R.A., A.J. Ferreira, and R.A. Dos Santos, Opportunities for targeting the angiotensin-converting enzyme 2/angiotensin-(1-7)/mas receptor pathway in hypertension. Curr Hypertens Rep, 2013. 15(1): p. 31-8.

65. Ferreira, A.J., et al., New cardiovascular and pulmonary therapeutic strategies based on the Angiotensin-converting enzyme 2/angiotensin-(1-7)/mas receptor axis. Int J Hypertens, 2012. 2012: p. 147825.

66. Ferreira, A.J., et al., Therapeutic implications of the vasoprotective axis of the reninangiotensin system in cardiovascular diseases. Hypertension, 2010. 55(2): p. 207-13.

67. Thatcher, S.E., et al., Angiotensin-converting enzyme 2 decreases formation and severity of angiotensin II-induced abdominal aortic aneurysms. Arterioscler Thromb Vasc Biol, 2014. 34(12): p. 2617-23.

68. Danser, A.H., Novel drugs targeting hypertension: renin inhibitors. J Cardiovasc Pharmacol, 2007. 50(2): p. 105-11.

69. Seto, S.W., et al., Aliskiren limits abdominal aortic aneurysm, ventricular hypertrophy and atherosclerosis in an apolipoprotein-E-deficient mouse model. Clin Sci (Lond), 2014. 127(2): p. 123-34.

70. Davis, V., et al., Matrix metalloproteinase-2 production and its binding to the matrix are increased in abdominal aortic aneurysms. Arterioscler Thromb Vasc Biol, 1998. 18(10): p. 1625-33.

71. Petrinec, D., et al., Doxycycline inhibition of aneurysmal degeneration in an elastaseinduced rat model of abdominal aortic aneurysm: preservation of aortic elastin associated with suppressed production of 92 kD gelatinase. J Vasc Surg, 1996. 23(2): p. 336-46. 
72. Prall, A.K., et al., Doxycycline in patients with abdominal aortic aneurysms and in mice: comparison of serum levels and effect on aneurysm growth in mice. J Vasc Surg, 2002. 35(5): p. 923-9.

73. Vinh, A., et al., A novel histone deacetylase inhibitor reduces abdominal aortic aneurysm formation in angiotensin II-infused apolipoprotein E-deficient mice. J Vasc Res, 2008. 45(2): p. 143-52.

74. Curci, J.A., et al., Pharmacologic suppression of experimental abdominal aortic aneurysms: acomparison of doxycycline and four chemically modified tetracyclines. $\mathrm{J}$ Vasc Surg, 1998. 28(6): p. 1082-93.

75. Xie, X., et al., Doxycycline does not influence established abdominal aortic aneurysms in angiotensin II-infused mice. PLoS One, 2012. 7(9): p. e46411.

76. Curci, J.A., et al., Preoperative treatment with doxycycline reduces aortic wall expression and activation of matrix metalloproteinases in patients with abdominal aortic aneurysms. J Vasc Surg, 2000. 31(2): p. 325-42.

77. Baxter, B.T., et al., Prolonged administration of doxycycline in patients with small asymptomatic abdominal aortic aneurysms: report of a prospective (Phase II) multicenter study. J Vasc Surg, 2002. 36(1): p. 1-12.

78. Mosorin, M., et al., Use of doxycycline to decrease the growth rate of abdominal aortic aneurysms: a randomized, double-blind, placebo-controlled pilot study. J Vasc Surg, 2001. 34(4): p. 606-10.

79. Lederle, F.A., et al., Prevalence and associations of abdominal aortic aneurysm detected through screening. Aneurysm Detection and Management (ADAM) Veterans Affairs Cooperative Study Group. Ann Intern Med, 1997. 126(6): p. 441-9.

80. Liu, J., et al., Mechanism of inhibition of matrix metalloproteinase-2 expression by doxycycline in human aortic smooth muscle cells. J Vasc Surg, 2003. 38(6): p. 137683.

81. Abdul-Hussien, H., et al., Doxycycline therapy for abdominal aneurysm: Improved proteolytic balance through reduced neutrophil content. J Vasc Surg, 2009. 49(3): p. 741-9.

82. Lindeman, J.H., et al., Clinical trial of doxycycline for matrix metalloproteinase-9 inhibition in patients with an abdominal aneurysm: doxycycline selectively depletes aortic wall neutrophils and cytotoxic T cells. Circulation, 2009. 119(16): p. 2209-16.

83. Meijer, C.A., et al., Doxycycline for stabilization of abdominal aortic aneurysms: a randomized trial. Ann Intern Med, 2013. 159(12): p. 815-23.

84. Dinarello, C.A., A clinical perspective of IL-1beta as the gatekeeper of inflammation. Eur J Immunol, 2011. 41(5): p. 1203-17. 
85. Johnston, W.F., et al., Genetic and pharmacologic disruption of interleukin-1beta signaling inhibits experimental aortic aneurysm formation. Arterioscler Thromb Vasc Biol, 2013. 33(2): p. 294-304.

86. Juvonen, J., et al., Elevated circulating levels of inflammatory cytokines in patients with abdominal aortic aneurysm. Arterioscler Thromb Vasc Biol, 1997. 17(11): p. 2843-7.

87. Pearce, W.H., et al., Interleukin-1 beta and tumor necrosis factor-alpha release in normal and diseased human infrarenal aortas. J Vasc Surg, 1992. 16(5): p. 784-9.

88. Vijaynagar, B., et al., Potential role for anti-angiogenic therapy in abdominal aortic aneurysms. Eur J Clin Invest, 2013. 43(7): p. 758-65.

89. Holmes, D.R., et al., Medial neovascularization in abdominal aortic aneurysms: a histopathologic marker of aneurysmal degeneration with pathophysiologic implications. J Vasc Surg, 1995. 21(5): p. 761-71; discussion 771-2.

90. Thompson, M.M., et al., Angiogenesis in abdominal aortic aneurysms. Eur J Vasc Endovasc Surg, 1996. 11(4): p. 464-9.

91. Kobayashi, M., et al., Expression of angiogenesis and angiogenic factors in human aortic vascular disease. J Surg Res, 2002. 106(2): p. 239-45.

92. Choke, E., et al., Vascular endothelial growth factor enhances angiotensin II-induced aneurysm formation in apolipoprotein E-deficient mice. J Vasc Surg, 2010. 52(1): p. 159-166 e1.

93. Paik, D.C., et al., Ongoing angiogenesis in blood vessels of the abdominal aortic aneurysm. Exp Mol Med, 2004. 36(6): p. 524-33.

94. Choke, E., et al., Abdominal aortic aneurysm rupture is associated with increased medial neovascularization and overexpression of proangiogenic cytokines. Arterioscler Thromb Vasc Biol, 2006. 26(9): p. 2077-82.

95. Ferrara, N., et al., Discovery and development of bevacizumab, an anti-VEGF antibody for treating cancer. Nat Rev Drug Discov, 2004. 3(5): p. 391-400.

96. Kaneko, H., et al., Role of vascular endothelial growth factor-A in development of abdominal aortic aneurysm. Cardiovasc Res, 2011. 91(2): p. 358-67.

97. Emeto, T.I., et al., Relevance of urocortins to cardiovascular disease. J Mol Cell Cardiol, 2011. 51(3): p. 299-307.

98. Walczewska, J., et al., The role of urocortins in the cardiovascular system. J Physiol Pharmacol, 2014. 65(6): p. 753-66.

99. Ng, L.L., et al., Plasma urocortin in human systolic heart failure. Clin Sci (Lond), 2004. 106(4): p. 383-8. 
100. Hasegawa, A., et al., Vasoprotective effects of urocortin 1 against atherosclerosis in vitro and in vivo. PLoS One, 2014. 9(12): p. e110866.

101. Emeto, T.I., et al., Urocortin 2 is associated with abdominal aortic aneurysm and mediates anti-proliferative effects on vascular smooth muscle cells via corticotrophin releasing factor receptor 2. Clin Sci (Lond), 2014. 126(7): p. 517-27.

102. Rush, C., et al., Whole genome expression analysis within the angiotensin IIapolipoprotein E deficient mouse model of abdominal aortic aneurysm. BMC Genomics, 2009. 10: p. 298.

103. Honjo, T., et al., Endothelial urocortin has potent antioxidative properties and is upregulated by inflammatory cytokines and pitavastatin. J Vasc Res, 2006. 43(2): p. 131-8.

104. Bale, T.L., et al., Corticotropin-releasing factor receptor 2 is a tonic suppressor of vascularization. Proc Natl Acad Sci U S A, 2002. 99(11): p. 7734-9. 